\title{
HMI conventions for process control graphics
}

\author{
Ruud N. Pikaar* \\ ErgoS Engineering \& Ergonomics, P.O. Box 267, NL 7500 AG, Enschede, The Netherlands
}

\begin{abstract}
Process operators supervise and control complex processes. To enable the operator to do an adequate job, instrumentation and process control engineers need to address several related topics, such as console design, information design, navigation, and alarm management. In process control upgrade projects, usually a 1:1 conversion of existing graphics is proposed. This paper suggests another approach, efficiently leading to a reduced number of new powerful process graphics, supported by a permanent process overview displays. In addition a road map for structuring content (process information) and conventions for the presentation of objects, symbols, and so on, has been developed. The impact of the human factors engineering approach on process control upgrade projects is illustrated by several cases.
\end{abstract}

Keywords: Process control, power graphic, permanent overview display

\section{Human Factors Engineering in control centers}

In process control upgrade projects, instrumentation and control software will be replaced. From a process control or instrument engineering point of view, usually a 1:1 conversion of existing graphics is proposed. From an Ergonomics or Human Factors Engineering (HFE) point of view, a 1:1 conversion is not likely to result into optimal operator task performance. In addition, the 1:1 conversion approach does not utilize the information display capabilities of modern visual display terminals, such as wide screen and large screen technology. For example, wide screens allow more process data to be displayed on one graphic ("picture" on the screen), than 15 year old low resolution displays. Thus, a larger part of a process unit and its connections may be visible to the operator enabling an easier understanding of the processes to control and supervise. In addition, this leads to less graphics to use, less graphics to build, and possibly less displays needed at the workplace [9]. Therefore, a contribution by ergonomists or HFE should be considered. The aim of ergonomics is to optimize the work system. In general terms, this requires a joint design of both social and technical aspects of the system [8]. Job design, operator work- load, control centre and workplace layout, instrumentation, information display, environment, and many more topics have to be addressed.

The Human Factors professional may not have much background in process control or other engineering sciences. Therefore, he relies on methodology, i.e. a systematic design approach. The HF professional tries to get insight in the relationships between relevant human factors, thus touching on work of the other disciplines within a project. For example, the process control engineer may be responsible for the contract with the instrumentation vendor, deciding on workplace equipment ('we need wide screen monitors'), and graphics ('use the process and instrumentation diagrams as a starting point'). Operations may think otherwise. Civil engineering may dictate workplace location because of available cabling ducts. Sometimes nobody takes responsibility for the design of operator workplaces.

The HFE may fill in the gaps between technical engineering disciplines, backed up by the systems ergonomics design approach of the ISO 11064 Ergonomic design of control centers series of standards [5]. Of course, a close cooperation between HFE and technical engineering disciplines will be needed.

\footnotetext{
* Corresponding author. Email: Ruud.Pikaar@ErgoS.nl
} 


\section{Case studies}

This paper is based on several cases of graphics upgrade projects, published by Pikaar et.al $[9,10]$. It should be noted that case studies have a methodological problem. Statistically valid comparisons on a project level cannot be made. A project is not carried out twice, i.e. with or without an ergonomics contribution. An ergonomics contribution usually starts with a task analysis of the existing situation $[5,8]$ to get sufficient insight in the process operators' tasks. In order to determine the impact of ergonomics, one should redo the task analysis after project completion. However, this is rarely possible in a commercial market.

The author strongly believes [8] that HFE experiences in industrial settings, which implies many practical constraints, should be reported in ergonomics literature notwithstanding the methodological problem of $\mathrm{N}=1$ ("a story about one case"). Publication is essential to bridge the gap between scientific research and practice. In the case of graphics, new ideas may be developed and tested against old presentation formats in a laboratory setting, with student volunteers (instead of experienced process technicians). The author did so many years ago [6], involving trainee operators in experimental set ups, and so did other researchers [11]. After several decades of experimenting, one might expect a lot of guidance on the design of process graphics. However, there are only a few guidelines available, covering the full scope of operator - process interaction (which will be discussed in section 4). There are still a number of unsolved questions. For example: what is to be considered too much, too little, or just the right amount of content (number of controlled variables) on a graphic? And, this is exactly the first question a process control engineer will ask. In conclusion, case studies may lead the way to finding the gap between scientific research and practice/applied ergonomics.

\section{Human Factors in control centers}

To enable process operators to do an adequate job, one needs to address at least four topics:

- workplace design - the operator console,

- process graphics,

- human-computer interaction - navigation and control,

- alarm management.
All topics are related to each other and therefore all are related to graphics design. Though important factors in control centre design, ventilation, acoustics, and light conditions will not be discussed here. Also job design or operator workload issues will not be discussed in this paper. Refer to [7] and [2] for ergonomics control room guidelines published by stakeholders (process industries, organized in the Instrument Users' associations (SIREP-WIB-EXERA) and the Materials Users' association (EEMUA)).

\subsection{Workplace design}

Workplace layout includes the instrumentation and equipment needed at the workplace and how it is organized. The number of screens on the desk and elsewhere (such as a large overview display) determines workplace measurements, and viewing distances. To design graphics, we need to know about viewing distances. Legibility of important text is guaranteed at a maximum of $200 \mathrm{x}$ character/ numerical height. For secondary text this may be $250 \mathrm{x}$ character height. At a viewing distance of $1 \mathrm{~m}$, this results into $5 \mathrm{~mm}$, respectively $4 \mathrm{~mm}$ character height. Legibility guidelines are based on the critical detail an average person (visual acuity: 1) is able to distinguish, i.e. one minute of arc or approximately $1 \mathrm{~mm}$ at $3 \mathrm{~m}$ distance. For engineers, this guideline is straight forward.

In practice, the simple question of viewing distance leads to different points of view. Predominantly in Europe, control room workplace designs are based on the operator being able to use information on up to 4 displays in a (curved) row. Typical viewing distances will be up to $1000 \mathrm{~mm}$. USA based guidelines assume the operator to be working with one screen out of many at a time, at short viewing distances of $600 \mathrm{~mm}$. Anyhow, console design influences character sizes, screen sizes, and graphics layout.

\subsection{Human computer interaction - Navigation}

Graphics are part of the operator interface of the process control system. The selection of graphics, detailed process data (controllers), alarm overview and other pictures is governed by interaction software. Depending on how it is organized, interaction requires space for a menu or control bar on the screen, usually repeated on each screen. If you want to have a one touch access to each graphic, a button is needed for each graphic. Other examples of data repeated on each screen are time and date, the last three alarm 
lines, and a vendor logo, being shown on each display. The number of graphics has an impact on interaction needed. Of course, this also relates to the number of screens on the console, ease of selecting and finding data, ease of switching between graphics and so on.

\subsection{Alarm management and process overview}

Complex processes require tools to present an overall view of the processes to be supervised and the status of off-normal situations (alarms). De Groot [4] takes a task oriented approach and developed powerful process overview displays for several applications. For multi operator situations, such displays are permanently available on large screens. He argues that, consequently, there is no need to present process overview data on the console based graphics.

Off-normal process information (commonly indicated as alarm) adds to the overview, improving operator awareness of the process conditions. In many cases an off-normal overview is presented on a separate display. Recent developments can be found in the ASM Guidelines for effective display design [1].

\subsection{Graphics design}

Graphics design requires insight in the workplace, instrument system characteristics, and the process operations philosophy. Current views on guidelines for graphics design are presented in section 4. Instrumentation systems on the market have different capabilities and limitations regarding information display. Therefore, it is possible that available character fonts, font sizes, and/or colors, do not match other workplace requirements. For example, if character sizes are relatively small, and cannot (easily) be changed, you will have to adapt the workplace layout, that is reduce the average viewing distances to the instrumentation displays. Usually instrumentation vendors have developed typicals (standard symbols and process variable presentation formats) that cannot be changed without compromising future upgrades (at least according to the vendor). Of course, this will not be a problem, if the typicals are in line with ergonomic guidelines and other user requirements. The authors' field experiences indicate this may not be the case.

Hence, it will be required to develop HMI Conventions for each typical instrumentation system and for each typical application (type of processes, industry, and company).

\section{Guidelines for process graphics design}

Every decade new design guidelines for process graphics on visual display units have been published. Besides several huge compilations for the nuclear power industries, practice oriented guidelines have been published by Gilmore et.al [3] in 1989, Pikaar et.al [7] in 1998, and recently by Bullemer et.al [1]. These guidelines hardly changed over the decades, which becomes evident comparing the guidelines of [7] and [1]. The guidelines by Pikaar et.al. have been developed independent from instrumentation system vendors and can be summarized as follows (for a full listing of guidelines refer to [7]):

\section{Picture hierarchy and navigation}

- Minimize hierarchy depth of graphics, three levels preferably being the maximum.

- Graphics structure should be transparent; navigation should be consistent and simple.

- Reduce the number of graphics and maximize information content of each graphic by careful use of coding, structuring, and labeling.

- Frequently used graphics should be directly accessible; a menu selection type of dialogue is less suitable.

\section{Content of graphics}

- Provide types of graphics that support the main operator activities process monitoring, diagnostic tasks, specific operations (start-up, production switch), and off-normal handling;

- Display task relevant information only, using overview graphics for key readings and control graphics for primary variables; secondary variables to be shown in overlays, pop-up windows, or detail graphics;

- Display information in an appropriate format;

- Avoid complex and cluttered pictures;

- Provide adequate identification of plant items and instruments.

\section{Layout of graphics}

- Ensure consistency by defining a general format and conventions for process flow direction(s), symbols, line crossings, location of process values relative to equipment, etc.

- Emphasize key items and related information by coding and structuring. 


\section{Coding and structuring of information}

- Avoid redundant information if there is no real, established, need to use redundancy;

- Structure information by (in order of preference): empty spaces, separation lines, grey levels, and color coding.

- Structure information by minimizing the number of left/right margins as well as horizontal lines, for text or numerical data items.

\section{Color coding}

- Minimize the number of colors used

- Consider color vision defects

- Consider color coding conventions (for example red=warning, amber=caution).

- Use a single, non distracting color for all picture backgrounds. The foreground color should be distinct against the chosen background color.

- Avoid high brightness colors at relatively large areas.

\section{Alpha-numeric information}

- Apply guidelines on character legibility, abbreviations, labeling, messages and numerical values.

Research related to the ASM Guidelines for effective display design $[1,11]$ is sponsored by several process industries and one major instrumentation vendor. A summary of guidelines:

- Display types; support a range of operator tasks and interactions through various display types.

- Display content; support all operator monitoring, troubleshooting, and control activities with all essential information.

- Display style; ensure that displays are not overly complex or cluttered due to the inappropriate use of full intensity colors and graphical details.

- Display layout; have a consistent arrangement of objects and information across similar displays that are appropriate to process behaviors.

- Navigation; facilitate quick direct access to primary displays and minimal keystrokes to secondary and associated displays.

- Use color to facilitate the discrimination of the most important information and convey the information consistently throughout the control room work environment.

- Symbols and Process connections are depicted in a meaningful and consistent manner.
- Text and numbers; information presented with text and numbers are legible and easily understood from the operator's typical position.

- Interactions with displays; input mechanisms are accessible with minimal keystrokes, and with appropriate error avoidance techniques.

Both sets of guidelines equally apply

- one design rule: keep it simple or, in case of existing graphics, simplify as much as possible,

- and one content rule: present only task related information.

Differences between both guidelines can be found in the level of simplification. At what level looses the trained operator the meaning of a symbol? A few examples may illustrate these questions, as well as point towards interesting areas for research (identifying a gap between science and applied ergonomics). For example, one could argue that the pump symbols in figure 1, taken from [1], is not a simple symbol.

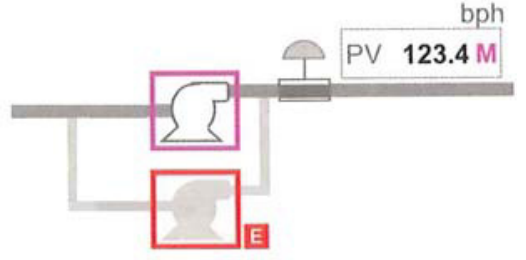

Figure 1. Pump symbols and Process Variable box.

This figure also illustrates another shortcoming of this particular software: a process variable cannot be shown without a box around it (top - left), and a reserved position for a status indicator, just behind the numerical value ("M").

Figure 2, taken from [9] shows a simplified symbol for a pump (circle with small arrow inside; colour may be used to indicate status, input and output "piping" are drawn on the same horizontal line). Also note a possible representation of 2 parallel pumps by characters " $\mathrm{A}$ " and " $\mathrm{B}$ "; the highlighted character indicates the running pump.

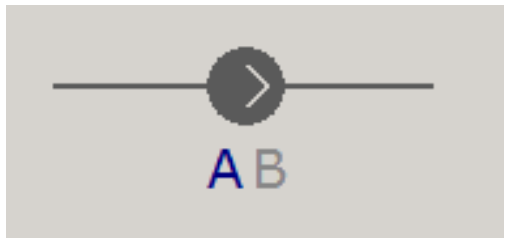

Figure 2. Simplified symbol for 2 parallel pumps. 
Another typical example of simplified symbols can be found for heat exchangers, as shown in figure 3.

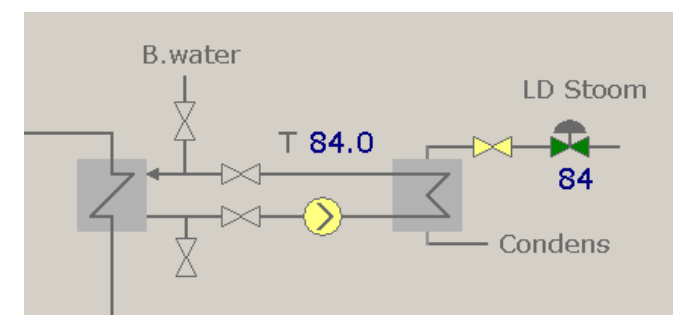

Figure 3. Examples of simplified heat exchanger symbols; two symbols are shown.

Process data may be displayed inside a vessel, or reactor symbol, instead of aside the component, leading to a more effective use of the graphics area.

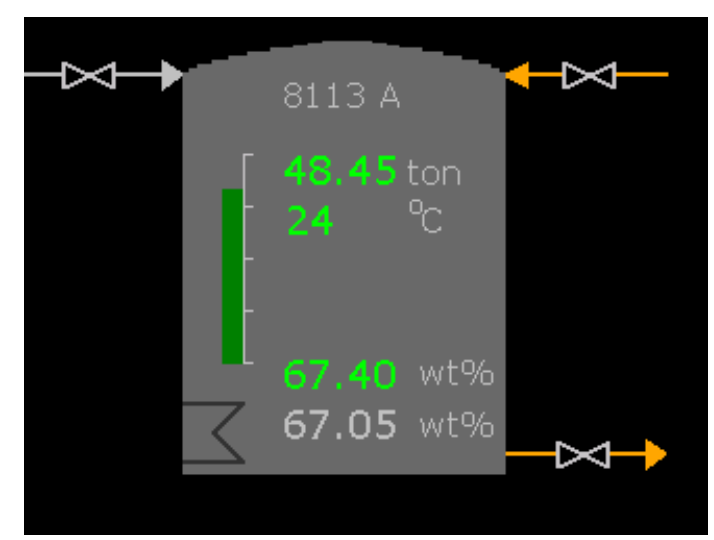

Figure 4. Process data inside tankage symbol.

\section{Operating Philosophy and HMI Conventions}

An Operating Philosophy describes what process operators are expected to do and the means required to do the job adequately (displays, controls, communication tools, and process data). Amongst others, process characteristics, the division of process data over graphics, the hierarchy of graphics, and the ease of navigating between process data, may determine the number of displays on the console.

A typical Operating Philosophy includes:

- an overview of process operator tasks, related to (different) operating conditions (start-up, offnormal, calamity, switch-over, turn around);
- determination of the critical number of graphics required to perform each task; leading question will be, how many screens do you need to be able to cope with situations for which a maximum of information is needed;

- sizes and viewing distances;

- the application of shared (overview) displays.

- alarm management philosophy (for example: separate alarm overview display or integrated in process graphics).

\section{Operating Philosophy - example}

Based on a summary of task analysis data, gathered by Pikaar and colleagues [6] over several decades of process control room projects the following generalized Operating Philosophy is recommended as a starting point for discussion or further development.

- The operator console will have two identical workplaces. One workplace will be used for normal process conditions. The other workplace will be available for an additional operator in case of off-normal conditions, trainee, and so on. In addition, redundancy of equipment can be guaranteed.

- The number of screens at one workplace will be limited to the number an operator can overlook in one glance. This means two "working screens" in the centre of the workplace, and one or two secondary displays to the left and right of the primary displays (working screens).

- Avoid a second row of displays, directly on top of the primary screens. Thus, the operator will be able to see other users of the control room, and have visual contact for direct and high quality communication. Also, it will be possible to locate (large) overview displays, shared with other control room workplaces.

- An essential feature is a shared process overview display. This display (or several screens) provides a permanent overview of key process variables. For example, normal process conditions of a waste incinerator line including flue gas treatment, can be supervised adequately by showing approximately $20-25$ selected process variables. This is possible because operators know a permanent display by heart. Therefore, the amount of static process details on such a display is limited. Another example: off-shore gas production platforms can be "summarized" in 5-10 key variables. Each line of the graphic 
shown in figure 5 (below) represents one production platform.

- Develop powerful graphics, emphasizing process functionality, rather than the mechanical design ("what does the unit look like") or topological details ("how is piping connected").

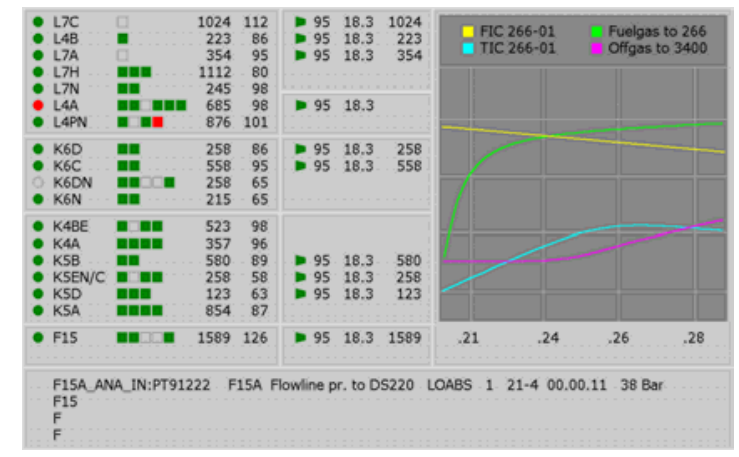

Figure 5. Example of a permanent process overview display.

\section{HMI Convention}

Using the capabilities of modern displays and software, such as the use of overlays, pop-up faceplates, or tool tips, up to 5 or 6 traditional process graphics can be combined into one so called Power Graphic. The key to Power Graphics is a set of rules for designing functional representations of process units and a standardized set of object symbols, simplified as compared to the usual symbols. The set of rules will be called the HMI Conventions and can be based on guidelines for process graphics as presented in section 4.

\section{Roadmap to Power Graphics}

Assuming an accepted operations philosophy and a HMI Conventions document, both tuned to the type of process units as well as selected instrumentation system (vendor), the following steps lead to Power Graphics.

1. Starting point: old/existing graphics (figure 6)

2. Reduce process variables /controllers to a process value only; other related data will be available in a pop-up, overlay, etc.

3. Remove redundant symbols, lines, crossing lines

4. Simplify symbols as much as possible; replace by symbols agreed upon in the HMI Conventions.

5. Check whether the graphic is self explanatory. You will need experienced operators to discuss.

6. Remove content not needed ("this pump has been removed years ago, they forgot to update the graphic"). Obviously, again you need contact with operators.

7. Check whether there is enough free space to combine this graphic with a related graphic. If so, do so, meanwhile removing (double) identification of connecting piping.

8. Simplify structure further.... Etc.
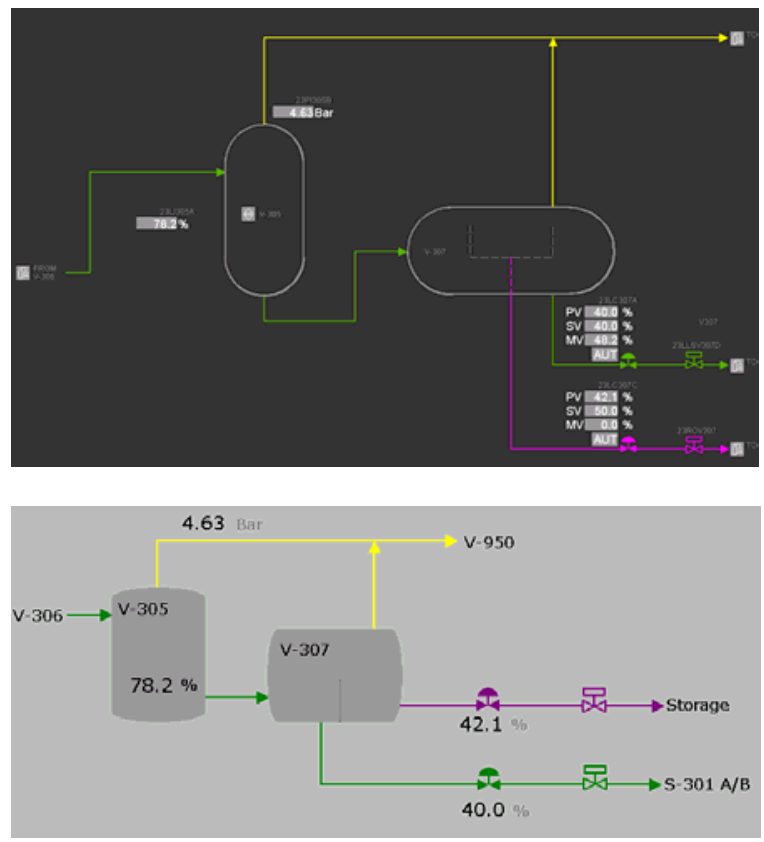

Figure 6. Top: original graphic, below: after first simplifications.

\section{Case studies}

The Operating Philosophy (section 5) has been applied in many process control room projects. These projects included all major instrumentation system vendors. In all cases, HMI Conventions (or style guides) have been developed adapted to the characteristics of the particular industry and instrumentation vendor, as well as examples of redesigned graphics In several cases, the project owner then took over further development and implementation. In other cases, the HFE played a major role in development and implementation of Power Graphics. Below, there is a summary of recently published cases $[9,10]$. 


\section{Case 1 - Food industry}

The project concerned the combination of three local control rooms for batch processing into a central control room, meanwhile upgrading the old instrumentation systems and adding a new process line. After a situation analysis in each control room, a working group of operator representatives was formed. The working group got an introduction in graphics design. One senior operator and the ergonomist redesigned 121 graphics, resulting into 40 Power Graphics, plus one overview graphic per plant.

As a strategy for change, first, the old graphics were converted 1:1. The Power Graphics were introduced as an intermediate level of graphics between the permanent process overview and the converted old graphics. All data of the existing graphics could be accessed on this level. After a period of time during which the operators could get accustomed to the new situation, the original graphics have been abandoned by almost all users.

\section{Case 2 - Chemical plant}

Three main processing lines were to be extended by a $4^{\text {th }}$ line. There is one control room operator. The situation analysis in the existing control room showed that this operator had a high workload. It was questionable whether the operator would also be able to handle the additional $4^{\text {th }}$ line. Finding related process variables was elaborate, because they were spread over several graphics. It was decided to redesign existing graphics for implementation in the new processing line. One operator per operator team participated in a working group. A workshop on ergonomic design guidelines was organized. Within a few weeks all basics for simplifying graphics were thoroughly understood and the working group took over redesign work almost completely and successfully from the ergonomist.

Instead of an estimated 55 graphics at a cost of $€ 1.000$ each, 20 graphics were developed. Graphics design was done by the working group members during quiet operation hours. Next, the DCS vendor implemented the graphics. The operators did the factory acceptance tests. Based on enthusiasm of the operators and the relatively low costs, it was decided to have all graphics redesigned, while upgrading the old system. A reduction from 200 to 70 Power Graphics could be achieved.

Because of the plant extension and an already high operator workload, a manpower increase of one operator per shift was planned. Because of the Power Graphics, operator workload was reduced. As a result, one operator now handles all processes during normal process conditions.

\section{Case 3 - On-shore control and supervision of} gas production

In the area of gas production, processes are relatively simple. The challenge lies in the number of production facilities, remote control, communication between control room and off-shore (un)manned sites, and dispatching tasks. Dispatching ("selling" gas) requires production flow control. For a company with approximately 40 off-shore assets in one area, onshore supervision had to be developed. Off-shore, 1800 graphics are in use (45 on average per asset). By systematically simplifying the existing graphics, discussing the use (or non-use) of each variable with experienced operators, a reduction of an estimated $80 \%$ could be achieved. In addition, a rationalization process took place (streamlining comparable units). Here, it proved to be worthwhile to develop HMI Conventions including standards on the level of processing units (such as a compressor unit), and components (valve, pump, vessel). An additional question arose regarding different instrumentation systems. In practice it is almost impossible to give them the same look and feel. Question is, whether this should be considered a problem.

\section{Conclusions and recommendations}

1. A situation (or task) analysis, usually reveals cluttered and ill structured data on 15 to 20 years old graphics. Ill structured data doesn't assist the operator in acquiring a good process awareness. Unplanned unit shut down or production losses have been reported during task analyses, because operators missed vital process information (it is not usual to report this type of events in literature). Usually, content (processes and equipment) changed over the years; graphics did not. Practical experiences of 1:1 conversion projects indicate (unexpected) problems due to ill documented process changes.

2. Old technology graphics can be considerably simplified, applying a set of straight forward, ergonomic guidelines on information display. A 50 to $80 \%$ reduction can be achieved, leading to an improvement of the operator task.

3. In addition a permanent process overview display proved to be a powerful operator tool. An 
overview typically consists of key performance indicators and most important process variables.

4. Graphics and interaction design have an impact on console design and vice versa. In addition, an improved display technology, in particular higher resolutions and wide screens, enable better representations of symbols, process equipment, indicators, etc. A 1:1 conversion doesn't utilize these advantages.

5. Graphics and interaction design have a major impact on operator workload. If it is difficult for one operator to get a good overview of the process status, the need may be felt to have two operator for the same process control job. If a task analysis shows that this is due to badly designed graphics, more cost effective solutions can be implemented.

6. Instrument upgrade project teams should consider a human factors inspired graphics redesign approach. The suggested approach includes an operator task analysis/ situation analysis, a review of existing graphics, and an analysis of the capabilities and limitations of the instrumentation system. Next, an Operating Philosophy and HMI Conventions have to be developed and agreed upon.

7. The first new graphics may be developed by ergonomists and discussed with operations, instrumentation engineers and vendor. Next, operators gradually could take over the development, assisted by the ergonomist with comments, suggestions, check on correct use of the style guide and so on. In any case, the input by experienced process operators is essential.

The human factors profession has the tools and knowledge to investigate workload issues, workplace design, information design, and knows how to organize user participation.

\section{Acknowledgement}

The author thanks all his colleagues at ErgoS Engineering and Ergonomics for their valuable contributions to this paper.

\section{References}

Copies of papers published by ErgoS Engineering \& Ergonomics can be requested at contact@ergos.nl.

[1] Bullemer, P. et.al. (2008), ASM Consortium Guidelines Effective Operator Display Design (Honeywell International Inc./ASM Consortium).

[2] EEMUA (2002); Process plant control desks utilising human-computer interface, a guide to design, operational an d human interface issues. Publication nr. 201.

[3] Gilmore, W.E., D.I. Gertman, H.S. Blackman (1989), UserComputer Interface in Process Control; Academic Press, San Diego.

[4] Groot, N. de, R.N. Pikaar (2006), Videowall Information Design: useless and useful applications. In: Meeting Diversity in ergonomics, Proceedings of IEA2006 (Elsevier, Amsterdam).

[5] ISO 11064 (1998-2007), Ergonomic Design of Control Centres - multi part standard. International Organization for Standardization (The engineering equipment and materials users'association, London).

[6] Pikaar, R.N. (1981); How people discover input/output relationships. In: H.G. Stasssen, Proceedings of the $1^{\text {st }}$ european annual conference on human decision making and manual control, Delft.

[7] Pikaar, R.N. et.al. (1998); Ergonomics in Process Control Rooms, Part 2: Design Guideline, (WIB International Instrument Users' Association, The Hague).

[8] Pikaar R.N. (2007), New challenges: Ergonomics in Engineering Projects. In: R.N. Pikaar, A.E.P. Koningsveld, \& P.J.M. Settels (eds.), Meeting Diversity in Ergonomics, (Elsevier, Amsterdam), 29-64.

[9] Pikaar R.N. (2010), Reduce graphics to improve Process overview. In: J. Wood, ICOCO 2010, International Control room design Conference, (Institute of Ergonomics and $\mathrm{Hu}-$ man Factors, Loughborough), 180-187.

[10] Pikaar, R.N., R. Landman, N. de Groot, L. de Graaf (2011); On-shore supervision of off-shore gas production - a human factors challenge. Accepted for publication in: Proceedings of the HFES-Europe Chapter meeting, Leeds (october 2011).

[11] Reising D.V., J. Laberge, P. Bullemer (2010), Supporting operator situation awareness with overview displays. In: J. Wood, ICOCO 2010, International Control room design Conference, (Institute of Ergonomics and Human Factors, Loughborough), 188-198. 